\title{
New Analysis of the Single Transverse-Spin Asymmetry for Hadron Production at RHIC
}

\author{
Koichi Kanazawa ${ }^{1}$ and Yuji Koike ${ }^{2}$ \\ ${ }^{1}$ Graduate School of Science and Technology, Niigata University, Ikarashi, Niigata 950-2181, Japan \\ ${ }^{2}$ Department of Physics, Niigata University, Ikarashi, Niigata 950-2181, Japan
}

\begin{abstract}
We analyze the single transverse-spin asymmetry (SSAs) for inclusive hadron production in the $p p$ collision at RHIC based on the twist-3 mechanism in QCD. As an origin of SSAs, we take into account of all kinds of pole contributions associated with the twist-3 quark-gluon correlation functions in the polarized proton. By the inclusion of the soft-fermion-pole contribution in addition to the soft-gluon-pole contribution, we find SSAs observed at RHIC are better described for all kinds of mesons. $P_{T}$-dependence of the asymmetry and the comparison with the FNAL data are also presented.
\end{abstract}




\section{Introduction}

Since the first observation of large single-spin asymmetry (SSA) in $p^{\uparrow} p \rightarrow \pi X$ by the FNAL-E704 Collaboration 11, SSA has been one of the hot topics in high energy spin physics. Large SSA has also been reported in semi-inclusive deep inelastic scattering (SIDIS), $e p^{\uparrow} \rightarrow e h X(h=\pi, K)$ 2. 3]. In the $p p$ collision, BNL-RHIC reported a similar large SSA at even higher energies [4, 5, 6, 7]. These large SSAs can not be explained within the conventional framework based on the parton model and perturbative QCD [8] which had been successful in describing various hard inclusive processes. They reflect novel quark-gluon substructure of hadrons beyond the parton model picture.

By now QCD mechanisms leading to such large SSA have been understood in some detail, and they are often classified into two categories depending on the kinematic regions they cover. One is the so-called "T-odd" distribution and fragmentation functions in the framework of the transverse momentum dependent (TMD) factorization. This mechanism describes the SSAs in the region of the small transverse momentum of the final hadron as a result of a correlation between the spin and the intrinsic transverse momentum of partons [9, 10, 11, 12, 13, 14, 15. Although this approach provides a physical interpretation of SSA, proof for the TMD factorization has been limited to the almostback-to-back jets production in $e^{+} e^{-}$annihilation [16, Drell-Yan [17] and SIDIS [18. In addition, the universality property of the TMD distribution function becomes unclear, in particular, for hadronhadron collisions [19. From the phenomenological analyses of SIDIS data given by CERN and DESY, a parametrization of the responsible TMD functions has been obtained 20 .

Another mechanism describes the SSA as a twist-3 effect in the framework of the collinear factorization $[21,22,23,24,25,26,27,28,29,30$. This mechanism is designed for the description of SSA in the region where the transverse momentum of the final hadron can be regarded as a hard scale in the process. In this mechanism twist-3 quark-gluon correlation functions replace the parton distribution and/or fragmentation functions, and gives the information on the multiparton correlations in the hadron. Although the above two mechanisms describe SSA in different kinematic regions, recent studies have shown that for a certain component contributing to SSA they give the equivalent description for SSA in the intermediate region of the transverse momentum for which both frameworks are valid, and these constitute a unique and unified QCD description for SSA 31.

In this paper we study SSA for the hadron $(\pi, K)$ productions in the $p p$ collision at the RHIC energies $(\sqrt{S}=200$ and $62.4 \mathrm{GeV})$,

$$
p^{\uparrow}\left(p, S_{\perp}\right)+p\left(p^{\prime}\right) \rightarrow h\left(P_{h}\right)+X,
$$

in the framework of the collinear factorization, extending the previous works $23,24,26,30$. Here $S_{\perp}$ represents the spin vector for the transversely polarized nucleon, and $p, p^{\prime}, P_{h}$ are the 4-momenta of each particle. The SSA for the above process is defined as $A_{N}=\left(\sigma^{\uparrow}-\sigma^{\downarrow}\right) /\left(\sigma^{\uparrow}+\sigma^{\downarrow}\right)$ where $\sigma^{\uparrow(\downarrow)}$ are the cross section for the process (11) corresponding to $S_{\perp}\left(-S_{\perp}\right)$. In this framework, three kinds of twist-3 cross section contribute to the single-spin-dependent cross section $\Delta \sigma \equiv \sigma^{\uparrow}-\sigma^{\downarrow}$ :

$$
\begin{aligned}
\Delta \sigma & =G^{(3)}\left(x_{1}, x_{2}\right) \otimes f\left(x^{\prime}\right) \otimes D(z) \otimes \hat{\sigma}_{A} \\
& +h(x) \otimes f\left(x^{\prime}\right) \otimes \widehat{E}^{(3)}\left(z_{1}, z_{2}\right) \otimes \hat{\sigma}_{B} \\
& +h(x) \otimes E^{(3)}\left(x_{1}^{\prime}, x_{2}^{\prime}\right) \otimes D(z) \otimes \hat{\sigma}_{C},
\end{aligned}
$$

where $\otimes$ denotes convolution with respect to the light-cone momentum fraction, and $f, h, D$ are, respectively, twist-2 unpolarized distribution, transversity distribution and the fragmentation function. Two variable functions with the upper index (3) are the twist-3 multiparton correlation functions: $G^{(3)}\left(x_{1}, x_{2}\right), E^{(3)}\left(x_{1}^{\prime}, x_{2}^{\prime}\right)$ and $\widehat{E}^{(3)}\left(z_{1}, z_{2}\right)$ are, respectively, the distribution function in the polarized nucleon, distribution function in the unpolarized nucleon and the twist-3 fragmentation function for the final hadron. $\hat{\sigma}_{A}, \hat{\sigma}_{B}, \hat{\sigma}_{C}$ are the perturbatively calculable partonic hard cross sections. It has been shown in 24 that the third term in (2) can be neglected compared to the first term because of the smallness of the partonic cross section; $\hat{\sigma}_{C} \ll \hat{\sigma}_{A}$. The second term of (2) involves the twist-3 fragmentation function, for which one needs different formalism to calculate the cross section 32 . In this circumstance, phenomenological analysis of the data has been performed including only the first term of (2) 23, 26]. According to the twist-3 formalism for SSA coming from the twist-3 distribution, the cross section occurs from a pole part of an internal propagator in the hard part (See e.g. 22, 25] for the 
detail of the formalism). These poles fix one of the momentum fractions in the twist-3 distribution, and those poles are classified into the soft-gluon-pole (SGP) and the soft-fermion-pole (SFP) corresponding, respectively, to the zero momentum of the gluonline and the quarkline in the twist-3 distribution. A characteristic feature of the SGP contribution is that some of them accompany a "derivative" of the SGP function, which enhances the cross section compared with the usual "nonderivative" terms. Accordingly it has been considered to be a main source of SSA compared with the SFP contribution, and the previous analyses of $A_{N}$ data focussed on the former [23, 26]. Even though the SFP contribution does not appear with the derivative, there is no clue that the corresponding nonperturbative function itself is small compared with the SGP function and therefore its importance depends on the magnitude of the partonic hard cross section. In a recent paper [30, one of the authors has shown that the partonic hard cross section for the SFP contribution is much larger than those for the SGP contribution, and the total SFP contribution could be a large source of SSA if the SFP function has a comparable magnitude as the SGP function.

The purpose of this paper is to present a numerical analysis of $A_{N}$ for the light-hadron production at RHIC, $p^{\uparrow} p \rightarrow h X(h=\pi, K)$, including all the effects from the twist-3 quark-gluon correlation functions contributing to the first term of (2). In principle, independent twist-3 quark-gluon distribution functions should be determined by performing a global analysis of various SSA data for SIDIS and the $p p$ collisions. However, SSA data obtained at HERMES and COMPASS are in the low $P_{T}$ region, and therefore is not suited for the analysis by the twist- 3 formalism. We thus focus on the asymmetry in the $p p$ collision in this analysis. Previous analysis of $A_{N}$ in the $p p$ collision included only the SGP contribution 23, 26, and could reproduce the $A_{N}$ obtained at FNAL and RHIC reasonably well, albeit with a deviation for $A_{N}^{K^{-}}$and the $P_{T}$ dependence 26, 5]. We will see in this paper that the SGP contribution itself is insufficient to understand the patterns shown in the RHIC $A_{N}$ data for the $\pi$ and $K$ production and inclusion of the SFP contribution significantly improves the agreement with the data. This suggests that the SFP contribution could be an important candidate for the origin of SSA.

The rest of the paper is organized as follows: In Sec 2, we summarize the necessary cross section formula as well as the twist-3 quark-gluon correlation functions. In Sec 3, we present our method of numerical analysis and the result. Section 4 is devoted to a brief summary.

\section{Cross-section formula}

There are two independent twist-3 quark-gluon correlation functions, $G_{F}$ and $\widetilde{G}_{F}$, contributing to the first term of (21). They are defined from the nucleon matrix element of the composite quark-gluon operator on the light-cone (See [25, 30] for the detail):

$$
\begin{aligned}
\int \frac{d \lambda}{2 \pi} \int \frac{d \mu}{2 \pi} e^{i \lambda x_{1}} e^{i \mu\left(x_{2}-x_{1}\right)}\left\langle p S_{\perp}\left|\bar{\psi}_{j}^{a}(0) g F^{\alpha \beta}(\mu n) n_{\beta} \psi_{i}^{a}(\lambda n)\right| p S_{\perp}\right\rangle \\
\quad=\frac{M_{N}}{4}(\not p)_{i j} \epsilon^{\alpha p n S_{\perp}} G_{F}^{a}\left(x_{1}, x_{2}\right)+i \frac{M_{N}}{4}\left(\gamma_{5} \not p\right)_{i j} S_{\perp}^{\alpha} \widetilde{G}_{F}^{a}\left(x_{1}, x_{2}\right)+\cdots
\end{aligned}
$$

where $n$ is a lightlike vector satisfying $p \cdot n=1, \psi_{i}^{a}$ is the quark field for flavor $a$ with the spinor index $i, F^{\alpha \beta}$ is the gluon's field strength, $\epsilon^{\alpha p n S_{\perp}} \equiv \epsilon^{\alpha \mu \nu \rho} p_{\mu} n_{\nu} S_{\perp \rho}\left(\epsilon_{0123} \equiv+1\right)$, and $\cdots$ denotes twist- 4 or higher. The nucleon mass $M_{N}$ is introduced to define $G_{F}$ and $\widetilde{G}_{F}$ dimensionless. From parity and $T$ invariance, $G_{F}^{a}\left(x_{1}, x_{2}\right)$ and $\widetilde{G}_{F}^{a}\left(x_{1}, x_{2}\right)$ satisfy the relation

$$
G_{F}^{a}\left(x_{1}, x_{2}\right)=G_{F}^{a}\left(x_{2}, x_{1}\right), \quad \widetilde{G}_{F}^{a}\left(x_{1}, x_{2}\right)=-\widetilde{G}_{F}^{a}\left(x_{2}, x_{1}\right) .
$$

The twist-3 correlation function for antiquark flavor can be defined similarly to (3), replacing the nonlocal operator in (3), $\bar{\psi}_{j}^{a}(0) g F^{\alpha \beta}(\mu n) n_{\beta} \psi_{i}^{a}(\lambda n)$, by its charge conjugated operator. The "antiquark" twist-3 correlation function obtained this way is related to the original "quark" correlation function as

$$
G_{F}^{\bar{a}}\left(x_{1}, x_{2}\right)=G_{F}^{a}\left(-x_{2},-x_{1}\right), \quad \widetilde{G}_{F}^{\bar{a}}\left(x_{1}, x_{2}\right)=-\widetilde{G}_{F}^{a}\left(-x_{2},-x_{1}\right) .
$$

The contribution to the single-spin-dependent cross section from the correlation function (3) has been obtained in the literature. For completeness we give the explicit formula here in our notation: The SGP 
contribution is given by 26

$$
\begin{aligned}
P_{h}^{0} \frac{d^{3} \Delta \sigma^{S G P}}{d P_{h}^{3}}= & \frac{\pi M_{N} \alpha_{S}^{2}}{S} \epsilon^{p n P_{h} S_{\perp}} \int_{z_{\min }}^{1} \frac{d z}{z^{3}} \int_{x_{\text {min }}^{\prime}}^{1} \frac{d x^{\prime}}{x^{\prime}} \int \frac{d x}{x} \frac{1}{x^{\prime} S+T / z} \delta\left(x-\frac{-x^{\prime} U / z}{x^{\prime} S+T / z}\right) \\
\times & \left(\frac{1}{-\hat{u}}\right)\left[\sum_{a, b, c}\left(G_{F}^{a}(x, x)-x \frac{d G_{F}^{a}(x, x)}{d x}\right) \times\right. \\
& \left\{f_{b}\left(x^{\prime}\right)\left(D_{c}(z) \hat{\sigma}_{a b \rightarrow c}^{G}+D_{\bar{c}}(z) \hat{\sigma}_{a b \rightarrow \bar{c}}^{G}\right)+f_{\bar{b}}\left(x^{\prime}\right)\left(D_{c}(z) \hat{\sigma}_{a \bar{b} \rightarrow c}^{G}+D_{\bar{c}}(z) \hat{\sigma}_{a \bar{b} \rightarrow \bar{c}}^{G}\right)\right\} \\
+ & \sum_{a}\left(G_{F}^{a}(x, x)-x \frac{d G_{F}^{a}(x, x)}{d x}\right) f_{\bar{a}}\left(x^{\prime}\right) D_{g}(z) \hat{\sigma}_{a \bar{a} \rightarrow g}^{G} \\
+ & \left.\sum_{a}\left(G_{F}^{a}(x, x)-x \frac{d G_{F}^{a}(x, x)}{d x}\right) G\left(x^{\prime}\right)\left(D_{a}(z) \hat{\sigma}_{a g \rightarrow a}^{G}+D_{g}(z) \hat{\sigma}_{a g \rightarrow g}^{G}\right)\right],
\end{aligned}
$$

where $\alpha_{s}=g^{2} /(4 \pi)$ is the strong coupling constant, the Mandelstam variables for the process are defined as

$$
\begin{aligned}
& S=\left(p+p^{\prime}\right)^{2}, \\
& T=\left(p-P_{h}\right)^{2}, \\
& U=\left(p^{\prime}-P_{h}\right)^{2},
\end{aligned}
$$

and the lower limits of the integration are given by $z_{\min }=-\frac{T+U}{S}, x_{\min }^{\prime}=\frac{-T / z}{S+U / z} \cdot f_{a}(x)$ and $D_{a}(z)$ are, respectively, distribution and fragmentation functions for quark (or antiquark) flavor $a$. $G(x)$ and $D_{g}(z)$ are, respectively, distribution and fragmentation functions for gluon. Partonic hard cross section in (6) are expressed in terms of the Mandelstam variables in the parton level:

$$
\begin{aligned}
\hat{s} & =\left(x p+x^{\prime} p^{\prime}\right)^{2}, \\
\hat{t} & =\left(x p-P_{h} / z\right)^{2}, \\
\hat{u} & =\left(x^{\prime} p^{\prime}-P_{h} / z\right)^{2},
\end{aligned}
$$

and they are given by

$$
\begin{aligned}
\hat{\sigma}_{a b \rightarrow c}^{G}= & {\left[\frac{1}{N_{c}^{2}}+\frac{1}{2 N_{c}^{2}}\left(1+\frac{\hat{u}}{\hat{t}}\right)\right]\left(\frac{\hat{s}^{2}+\hat{u}^{2}}{\hat{t}^{2}}\right) \delta_{a c} } \\
+ & {\left[\frac{1}{N_{c}^{2}}-\frac{N_{c}^{2}-2}{2 N_{c}^{2}}\left(1+\frac{\hat{u}}{\hat{t}}\right)\right]\left(\frac{\hat{s}^{2}+\hat{t}^{2}}{\hat{u}^{2}}\right) \delta_{b c} } \\
+ & {\left[-\frac{N_{c}^{2}+1}{N_{c}^{3}}-\frac{1}{N_{c}^{3}}\left(1+\frac{\hat{u}}{\hat{t}}\right)\right]\left(\frac{\hat{s}^{2}}{\hat{t} \hat{u}}\right) \delta_{a c} \delta_{b c}, } \\
\hat{\sigma}_{a b \rightarrow \bar{c}}^{G}= & 0, \\
\hat{\sigma}_{a \bar{b} \rightarrow c}^{G}= & {\left[\frac{N_{c}^{2}-2}{2 N_{c}^{2}}+\frac{1}{2 N_{c}^{2}}\left(1+\frac{\hat{u}}{\hat{t}}\right)\right]\left(\frac{\hat{s}^{2}+\hat{u}^{2}}{\hat{t}^{2}}\right) \delta_{a c} } \\
+ & {\left[-\frac{1}{2 N_{c}^{2}}-\frac{N_{c}^{2}-2}{2 N_{c}^{2}}\left(1+\frac{\hat{u}}{\hat{t}}\right)\right]\left(\frac{\hat{t} \hat{t}^{2}+\hat{u}^{2}}{\hat{s}^{2}}\right) \delta_{a b} } \\
+ & {\left[-\frac{1}{N_{c}^{3}}-\frac{1}{N_{c}^{3}}\left(1+\frac{\hat{u}}{\hat{t}}\right)\right]\left(\frac{\hat{u}^{2}}{\hat{\hat{s}} \hat{t}}\right) \delta_{a c} \delta_{b c}, } \\
\hat{\sigma}_{a \bar{b} \rightarrow \bar{c}}^{G}= & {\left[\frac{N_{c}^{2}-2}{2 N_{c}^{2}}-\frac{1}{N_{c}^{2}}\left(1+\frac{\hat{u}}{\hat{t}}\right)\right]\left(\frac{\hat{s}^{2}+\hat{t}^{2}}{\hat{u}^{2}}\right) \delta_{b c} } \\
+ & {\left[-\frac{1}{2 N_{c}^{2}}-\frac{1}{N_{c}^{2}}\left(1+\frac{\hat{u}}{\hat{t}}\right)\right]\left(\frac{\hat{t}^{2}+\hat{u}^{2}}{\hat{s}^{2}}\right) \delta_{a b} } \\
+ & {\left[-\frac{1}{N_{c}^{3}}-\frac{1}{N_{c}^{3}}\left(1+\frac{\hat{u}}{\hat{t}}\right)\right]\left(\frac{\hat{t}^{2}}{\hat{s} \hat{u}}\right) \delta_{a c} \delta_{b c}, } \\
\hat{\sigma}_{a \bar{a} \rightarrow g}^{G}= & \frac{1}{2 N_{c}^{3}}\left(\frac{\hat{t}}{\hat{u}}+\frac{\hat{u}}{\hat{t}}\right)\left(1+2 N_{c}^{2} \frac{\hat{t} \hat{u}}{\hat{s}^{2}}\right)
\end{aligned}
$$




$$
\begin{aligned}
& +\frac{1}{2 N_{c}}\left(1+\frac{\hat{u}}{\hat{t}}\right)\left(\frac{\hat{t}}{\hat{u}}+\frac{\hat{u}}{\hat{t}}\right)\left(1-N_{c}^{2} \frac{\hat{u}^{2}}{\hat{s}^{2}}\right), \\
\hat{\sigma}_{a g \rightarrow a}^{G} & =\frac{1}{2\left(N_{c}^{2}-1\right)}\left(\frac{\hat{s}}{\hat{u}}+\frac{\hat{u}}{\hat{s}}\right)\left(1-N_{c}^{2} \frac{\hat{u}^{2}}{\hat{t}^{2}}\right) \\
& +\frac{1}{2 N_{c}^{2}\left(N_{c}^{2}-1\right)}\left(1+\frac{\hat{u}}{\hat{t}}\right)\left(\frac{\hat{s}}{\hat{u}}+\frac{\hat{u}}{\hat{s}}\right)\left(1+2 N_{c}^{2} \frac{\hat{s} \hat{u}}{\hat{t}^{2}}\right), \\
\hat{\sigma}_{a g \rightarrow g}^{G} & =\frac{1}{2\left(N_{c}^{2}-1\right)}\left(\frac{\hat{s}}{\hat{t}}+\frac{\hat{t}}{\hat{s}}\right)\left(1-N_{c}^{2} \frac{\hat{t}^{2}}{\hat{u}^{2}}\right) \\
& +\frac{-1}{2\left(N_{c}^{2}-1\right)}\left(1+\frac{\hat{u}}{\hat{t}}\right)\left(\frac{\hat{s}}{\hat{t}}+\frac{\hat{t}}{\hat{s}}\right)\left(1-N_{c}^{2} \frac{\hat{s}^{2}}{\hat{u}^{2}}\right),
\end{aligned}
$$

where $N_{c}=3$ is the number of colors for SU(3). In (6), $\widetilde{G}_{F}^{a}$ does not contribute to the SGP contribution because $\widetilde{G}_{F}^{a}(x, x)=0$ due to the symmetry property (4), and $G_{F}^{a}(x, x)$ appears in the form of $\frac{d G_{F}^{a}(x, x)}{d x}-$ $G_{F}^{a}(x, x)[26,28$. The SFP contribution was obtained in [30] as

$$
\begin{aligned}
P_{h}^{0} \frac{d^{3} \Delta \sigma^{S F P}}{d P_{h}^{3}}= & \frac{\alpha_{S}^{2}}{S} \frac{M_{N} \pi}{2} \epsilon^{p n P_{h} S_{\perp}} \int_{z_{\min }}^{1} \frac{d z}{z^{3}} \int_{x_{m i n}^{\prime}}^{1} \frac{d x^{\prime}}{x^{\prime}} \int \frac{d x}{x} \frac{1}{x^{\prime} S+T / z} \delta\left(x-\frac{-x^{\prime} U / z}{x^{\prime} S+T / z}\right) \\
\times & {\left[\sum_{a, b, c}\left(G_{F}^{a}(0, x)+\widetilde{G}_{F}^{a}(0, x)\right) \times\right.} \\
& \left\{f_{b}\left(x^{\prime}\right)\left(D_{c}(z) \hat{\sigma}_{a b \rightarrow c}^{F}+D_{\bar{c}}(z) \hat{\sigma}_{a b \rightarrow \bar{c}}^{F}\right)+f_{\bar{b}}\left(x^{\prime}\right)\left(D_{c}(z) \hat{\sigma}_{a \bar{b} \rightarrow c}^{F}+D_{\bar{c}}(z) \hat{\sigma}_{a \bar{b} \rightarrow \bar{c}}^{F}\right)\right\} \\
+ & \sum_{a, b}\left(G_{F}^{a}(0, x)+\widetilde{G}_{F}^{a}(0, x)\right)\left(f_{b}\left(x^{\prime}\right) D_{g}(z) \hat{\sigma}_{a b \rightarrow g}^{F}+f_{\bar{b}}\left(x^{\prime}\right) D_{g}(z) \hat{\sigma}_{a \bar{b} \rightarrow g}^{F}\right) \\
+ & \sum_{a, c}\left(G_{F}^{a}(0, x)+\widetilde{G}_{F}^{a}(0, x)\right) G\left(x^{\prime}\right)\left(D_{c}(z) \hat{\sigma}_{a g \rightarrow c}^{F}+D_{\bar{c}}(z) \hat{\sigma}_{a g \rightarrow \bar{c}}^{F}\right) \\
+ & \left.\sum_{a}\left(G_{F}^{a}(0, x)+\widetilde{G}_{F}^{a}(0, x)\right) G\left(x^{\prime}\right) D_{g}(z) \hat{\sigma}_{a g \rightarrow g}^{F}\right],
\end{aligned}
$$

where the partonic hard cross sections are given by

$$
\begin{aligned}
\hat{\sigma}_{a b \rightarrow c}^{F}= & \frac{-\left(N_{c}^{2} \hat{s}+2 \hat{t}\right)\left(\hat{s}^{2}+\hat{u}^{2}\right)}{N_{c}^{2} \hat{t}^{3} \hat{u}} \delta_{a c}+\frac{-\left(N_{c}^{2} \hat{t}+\hat{u}-\hat{s}\right) \hat{s}}{N_{c}^{3} \hat{t} \hat{u}^{2}} \delta_{a b} \delta_{a c} \\
\hat{\sigma}_{a b \rightarrow \bar{c}}^{F}= & 0 \\
\hat{\sigma}_{a \bar{b} \rightarrow c}^{F}= & \frac{\left(N_{c}^{2} \hat{u}+2 \hat{t}\right)\left(\hat{s}^{2}+\hat{u}^{2}\right)}{N_{c}^{2} \hat{t}^{3} \hat{u}} \delta_{a c}+\frac{\left(N_{c}^{2} \hat{u}+2 \hat{s}\right)\left(\hat{t}^{2}+\hat{u}^{2}\right)}{N_{c}^{2} \hat{s}^{2} \hat{t} \hat{u}} \delta_{a b}-\frac{\left(N_{c}^{2}-1\right) \hat{u}^{2}}{N_{c}^{3} \hat{s} \hat{t}^{2}} \delta_{a b} \delta_{a c} \\
\hat{\sigma}_{a \bar{b} \rightarrow \bar{c}}^{F}= & \frac{-\left(N_{c}^{2} \hat{t}+2 \hat{s}\right)\left(\hat{t}^{2}+\hat{u}^{2}\right)}{N_{c}^{2} \hat{s}^{2} \hat{t} \hat{u}} \delta_{a b}+\frac{-N_{c}^{2} \hat{s}+\hat{t}-\hat{u}}{N_{c}^{3} \hat{u}^{2}} \delta_{a b} \delta_{a c} \\
\hat{\sigma}_{a b \rightarrow g}^{F}= & \frac{\left(N_{c}^{2} \hat{s}+2 \hat{t}\right)\left(\hat{s}^{2}+\hat{u}^{2}\right)}{N_{c}^{2} \hat{t}^{3} \hat{u}}+\frac{-1}{N_{c}^{3} \hat{s} \hat{u}^{2}}\left(N_{c}^{2}\left(\hat{s}^{3}+3 \hat{s}^{2} \hat{u}-2 \hat{u}^{3}\right)+\hat{s}^{3}-\hat{s}^{2} \hat{u}\right) \delta_{a b} \\
\hat{\sigma}_{a \bar{b} \rightarrow g}^{F}= & \frac{-\left(N_{c}^{2} \hat{u}+2 \hat{t}\right)\left(\hat{s}^{2}+\hat{u}^{2}\right)}{N_{c}^{2} \hat{t}^{3} \hat{u}} \\
+ & {\left[\frac{1}{N_{c}^{3}}\left(\frac{\hat{u}}{\hat{s} \hat{t}}+\frac{1}{\hat{u}}\right)+\frac{1}{N_{c}}\left(\frac{\hat{s}^{2}+\hat{s} \hat{t}+\hat{t}^{2}}{\hat{s} \hat{u}^{2}}-\frac{\hat{u}}{\hat{t}^{2}}\right)+\frac{N_{c}\left(\hat{u}^{3}-\hat{t}^{3}\right)\left(\hat{t}^{2}+\hat{u}^{2}\right)}{\hat{s}^{2} \hat{t}^{2} \hat{u}^{2}}\right] \delta_{a b} } \\
\hat{\sigma}_{a g \rightarrow c}^{F}= & {\left[\frac{N_{c}^{2}\left(\hat{s}^{3}-\hat{u}^{3}\right)\left(\hat{s}^{2}+\hat{u}^{2}\right)}{\left(N_{c}^{2}-1\right) \hat{s} \hat{t}^{3} \hat{u}^{2}}+\frac{\hat{s} \hat{u}\left(\hat{s}^{2}+\hat{s} \hat{u}-\hat{u}^{2}\right)-N_{c}^{2}\left(\hat{s}^{4}+\hat{s}^{3} \hat{u}+\hat{s}^{2} \hat{u}^{2}+\hat{s} \hat{u}^{3}+\hat{u}^{4}\right)}{N_{c}^{2}\left(N_{c}^{2}-1\right) \hat{s}^{2} \hat{u}^{2}}\right] \delta_{a c} } \\
+ & \frac{\left(N_{c}^{2} \hat{u}+2 \hat{s}\right)\left(\hat{t}^{2}+\hat{u}^{2}\right)}{N_{c}\left(N_{c}^{2}-1\right) \hat{s}^{2} \hat{t} \hat{u}} \\
\hat{\sigma}_{a g \rightarrow \bar{c}}^{F}= & \frac{\hat{s}+2 \hat{t}-N_{c}^{2} \hat{s}}{N_{c}^{2}\left(N_{c}^{2}-1\right) \hat{u}^{2}} \delta_{a c}+\frac{-\left(N_{c}^{2} \hat{t}+2 \hat{s}\right)\left(\hat{t}^{2}+\hat{u}^{2}\right)}{N_{c}\left(N_{c}^{2}-1\right) \hat{s}^{2} \hat{t} \hat{u}}
\end{aligned}
$$




$$
\begin{aligned}
\hat{\sigma}_{a g \rightarrow g}^{F} & =\frac{-N_{c}^{2}}{\left(N_{c}^{2}-1\right) \hat{s}^{2} \hat{t}^{3} \hat{u}^{2}}\left(4 \hat{s}^{6}+11 \hat{s}^{5} \hat{t}+19 \hat{s}^{4} \hat{t}^{2}+22 \hat{s}^{3} \hat{t}^{3}+19 \hat{s}^{2} \hat{t}^{4}+11 \hat{s} \hat{t}^{5}+4 \hat{t}^{6}\right) \\
& +\frac{1}{N_{c}^{2}\left(N_{c}^{2}-1\right) \hat{s}^{2} \hat{u}^{2}}\left(-\hat{s} \hat{t} \hat{u}^{2}+N_{c}^{2}\left(\hat{s}^{4}+\hat{s}^{3} \hat{t}+2 \hat{s}^{2} \hat{t}^{2}+\hat{s} \hat{t}^{3}+\hat{t}^{4}\right)\right) .
\end{aligned}
$$

The summation $\sum_{a, b, c}, \sum_{a, b}, \sum_{a}$ in (6) and (14) indicate that the sum of $a$ is over all quark and antiquark flavors, and the sum of $b$ and $c$ is over all quark flavors for the quark flavor $a$ and is over all antiquark flavors for the antiquark flavor $a$. As is shown in (14), the SFP contribution appears in the form of $G_{F}^{a}(0, x)+\widetilde{G}_{F}^{a}(0, x)$, which allows us to parametrize $G_{F}^{a}(0, x)+\widetilde{G}_{F}^{a}(0, x)$ when we study the process $p^{\uparrow} p \rightarrow h X$.

For completeness we also list the twist-2 unpolarized cross section for $p p \rightarrow h X[33$. which becomes the denominator of $A_{N}$ :

$$
\begin{aligned}
& P_{h}^{0} \frac{d^{3} \sigma^{\text {unpol }}}{d P_{h}^{3}}=\frac{\alpha_{S}^{2}}{S} \int_{z_{\min }}^{1} \frac{d z}{z^{2}} \int_{x_{\min }^{\prime}}^{1} \frac{d x^{\prime}}{x^{\prime}} \int \frac{d x}{x} \frac{1}{x^{\prime} S+T / z} \delta\left(x-\frac{-x^{\prime} U / z}{x^{\prime} S+T / z}\right) \\
& \times \quad\left[\sum _ { a , b , c } \left\{f_{a}(x) f_{b}\left(x^{\prime}\right)\left(D_{c}(z) \hat{\sigma}_{a b \rightarrow c}^{U}+D_{\bar{c}}(z) \hat{\sigma}_{a b \rightarrow \bar{c}}^{U}\right)\right.\right. \\
& \left.+f_{a}(x) f_{\bar{b}}\left(x^{\prime}\right)\left(D_{c}(z) \hat{\sigma}_{a \bar{b} \rightarrow c}^{U}+D_{\bar{c}}(z) \hat{\sigma}_{a \bar{b} \rightarrow \bar{c}}^{U}\right)\right\} \\
& +\sum_{a} f_{a}(x) f_{\bar{a}}\left(x^{\prime}\right) D_{g}(z) \hat{\sigma}_{a \bar{a} \rightarrow g}^{U} \\
& +\sum_{a} f_{a}(x) G\left(x^{\prime}\right)\left(D_{a}(z) \hat{\sigma}_{a g \rightarrow a}^{U}+D_{g}(z) \hat{\sigma}_{a g \rightarrow g}^{U}\right) \\
& +\sum_{a} G(x) f_{a}\left(x^{\prime}\right)\left(D_{a}(z) \hat{\sigma}_{g a \rightarrow a}^{U}+D_{g}(z) \hat{\sigma}_{g a \rightarrow g}^{U}\right) \\
& \left.+\sum_{a} G(x) G\left(x^{\prime}\right)\left(D_{g}(z) \hat{\sigma}_{g g \rightarrow g}^{U}+D_{a}(z) \hat{\sigma}_{g g \rightarrow a}^{U}\right)\right], \\
& \hat{\sigma}_{a b \rightarrow c}^{U}=\frac{C_{F}}{N_{c}} \frac{\hat{s}^{2}+\hat{u}^{2}}{\hat{t}^{2}} \delta_{a c}+\frac{C_{F}}{N_{c}} \frac{\hat{s}^{2}+\hat{t}^{2}}{\hat{u}^{2}} \delta_{b c}-\frac{2 C_{F}}{N_{c}^{2}} \frac{\hat{s}^{2}}{\hat{t} \hat{u}} \delta_{a c} \delta_{b c}, \\
& \hat{\sigma}_{a b \rightarrow \bar{c}}^{U}=0, \\
& \hat{\sigma}_{a \bar{b} \rightarrow c}^{U}=\frac{C_{F}}{N_{c}} \frac{\hat{t}^{2}+\hat{u}^{2}}{\hat{s}^{2}} \delta_{a b}+\frac{C_{F}}{N_{c}} \frac{\hat{s}^{2}+\hat{u}^{2}}{\hat{t}^{2}} \delta_{a c}-\frac{2 C_{F}}{N_{c}^{2}} \frac{\hat{u}^{2}}{\hat{s} \hat{t}} \delta_{a c} \delta_{b c}, \\
& \hat{\sigma}_{a \bar{b} \rightarrow \bar{c}}^{U}=\frac{C_{F}}{N_{c}} \frac{\hat{t}^{2}+\hat{u}^{2}}{\hat{s}^{2}} \delta_{a b}+\frac{C_{F}}{N_{c}} \frac{\hat{s}^{2}+\hat{t}^{2}}{\hat{u}^{2}} \delta_{b c}-\frac{2 C_{F}}{N_{c}^{2}} \frac{\hat{t}^{2}}{\hat{s} \hat{u}} \delta_{a c} \delta_{b c}, \\
& \hat{\sigma}_{a \bar{a} \rightarrow g}^{U}=\frac{2 C_{F}^{2}}{N_{c}} \frac{\hat{u}^{2}+\hat{t}^{2}}{\hat{u} \hat{t}}-2 C_{F} \frac{\hat{u}^{2}+\hat{t}^{2}}{\hat{s}^{2}}, \\
& \hat{\sigma}_{a g \rightarrow a}^{U}=-\frac{C_{F}}{N_{c}} \frac{\hat{u}^{2}+\hat{s}^{2}}{\hat{u} \hat{s}}+\frac{\hat{u}^{2}+\hat{s}^{2}}{\hat{t}^{2}}, \\
& \hat{\sigma}_{a g \rightarrow g}^{U}=-\frac{C_{F}}{N_{c}} \frac{\hat{s}^{2}+\hat{t}^{2}}{\hat{s} \hat{t}}+\frac{\hat{s}^{2}+\hat{t}^{2}}{\hat{u}^{2}}, \\
& \hat{\sigma}_{g a \rightarrow a}^{U}=\hat{\sigma}_{a g \rightarrow g}^{U} \\
& \hat{\sigma}_{g a \rightarrow g}^{U}=\hat{\sigma}_{a g \rightarrow a}^{U} \\
& \hat{\sigma}_{g g \rightarrow a}^{U}=\frac{1}{2 N_{c}} \frac{\hat{u}^{2}+\hat{t}^{2}}{\hat{u} \hat{t}}-\frac{1}{2 C_{F}} \frac{\hat{u}^{2}+\hat{t}^{2}}{\hat{s}^{2}}, \\
& \hat{\sigma}_{g g \rightarrow g}^{U}=\frac{9}{2}\left(3-\frac{\hat{u} \hat{t}}{\hat{s}^{2}}-\frac{\hat{u} \hat{s}}{\hat{t}^{2}}-\frac{\hat{s} \hat{t}}{\hat{u}^{2}}\right),
\end{aligned}
$$

where $C_{F}=\left(N_{c}^{2}-1\right) /\left(2 N_{c}\right)$ with $N_{c}=3$. With the above defined cross section, $A_{N}$ for $p^{\uparrow} p \rightarrow h X$ 
$(h=\pi, K)$ is given by

$$
A_{N}=\frac{P_{h}^{0} \frac{d^{3} \Delta \sigma^{S G P}}{d P_{h}^{3}}+P_{h}^{0} \frac{d^{3} \Delta \sigma^{S F P}}{d P_{h}^{3}}}{P_{h}^{0} \frac{d^{3} \sigma^{\text {unpol }}}{d P_{h}^{3}}} .
$$

\section{$3 \quad$ Numerical analysis}

Independent unknown functions appearing in $A_{N}$ are the SGP functions

$$
G_{F}^{a}(x, x),
$$

and the SFP functions

$$
G_{F}^{a}(0, x)+\widetilde{G}_{F}^{a}(0, x),
$$

for each quark and antiquark flavor $(a=u, d, s, \bar{u}, \bar{d}, \bar{s})$. Until now, the $A_{N}$ data obtained at RHIC are the only ones to which twist- 3 mechanism in the collinear factorization can be directly applied. In fact, the next-to-leading-order (NLO) QCD can describe the unpolarized cross section for the $p p \rightarrow \pi X$ process at RHIC energy, which constitutes the denominator of $A_{N}$. We thus use the $A_{N}$ data by the STAR Collaboration at $\sqrt{S}=200 \mathrm{GeV}$, 4, 5] and those by the BRAHMS Collaboration at $\sqrt{S}=62.4$ $\mathrm{GeV}$ [7]. All data for $A_{N}^{\pi^{0}}$ from the STAR Collaboration are in the range of $P_{h T} \geq 1 \mathrm{GeV}$, so we adopt all of the data presented in [4, 5] in the fitting. On the other hand, some of the BRAHMS data are in the low $P_{h T}$ region. But they reported data for $\pi^{ \pm}$and $K^{ \pm}$, which are important for disentangling the flavor structure of the quark-gluon correlations in the nucleon. So we will adopt data for $\pi^{ \pm}$and $K^{ \pm}$in [7] with $P_{h T} \geq 0.7 \mathrm{GeV}$ in the fitting for this initial study on the SFP contribution. The total number of data points turn out to be 61. Unlike the analysis in [26, we shall not use FNAL-E704 data in the fitting, since the NLO QCD can not reproduce the unpolarized cross section at the fixed target energy [34].

For the parametrization of the SGP and SFP functions, we assume the following form:

$$
\begin{aligned}
& G_{F}^{a}(x, x)=N_{a}^{G} x^{\alpha_{a}^{G}}(1-x)^{\beta_{a}^{G}} f_{a}(x), \\
& G_{F}^{a}(0, x)+\widetilde{G}_{F}^{a}(0, x)=N_{a}^{F} x^{\alpha_{a}^{F}}(1-x)^{\beta_{a}^{F}} f_{a}(x),
\end{aligned}
$$

where $f_{a}(x)$ is the unpolarized quark distribution, and $N_{a}^{G}, \alpha_{a}^{G}, \beta_{a}^{G}, N_{a}^{F}, \alpha_{a}^{F}, \beta_{a}^{F}$ are the parameters to be determined by the $\chi^{2}$ fitting to the data. Of course, the twist-3 quark-gluon correlation functions $G_{F}\left(x_{1}, x_{2}, \mu\right)$ and $\widetilde{G}_{F}\left(x_{1}, x_{2}, \mu\right)$ have their own logarithmic scale dependence ( $\mu$ dependence) which differs from that of the twist-2 unpolarized parton densities 1 and therefore the above ansatz for the SGP and SFP functions is not correct in the rigorous sense. However, at the present stage the SSA data is not so accurate, as the precise scale dependence of the twist-3 correlation is required. We therefore assume that the SGP and SFP component of the twist-3 correlation functions obey the same scale dependence as the twist-2 distribution through the ansatz (39) and (40) for simplicity. For the unpolarized parton densities in the nucleon, we use the LO parametrization of GRV98 [36]. As for the fragmentation functions for pions and kaons, we use the parametrization in [37] (hereafter referred to as the DSS fragmentation function), which can describe a greater variety of data in wider kinematic ranges, in particular for kaons, compared with the older more common ones in the literature. When we calculate $A_{N}$ we set $\mu=P_{h T}$ for the STAR data and $\mu=1 \mathrm{GeV}$ for the BRAHMS data in all distribution and fragmentation functions. In the $\chi^{2}$-fitting analysis below, we have used MINUIT package program in the CERN library.

Since our main purpose here is to investigate the role and importance of the SFP contribution in comparison to the SGP contribution, we will analyze the data in terms of the following three sets of the fitting functions:

FIT 1: SGP + SFP contributions with 13 free parameters:

$$
N_{u}^{G}, \quad N_{d}^{G}, \quad N_{s}^{G}=N_{\bar{s}}^{G}, \quad N_{\bar{u}}^{G}=N_{\bar{d}}^{G},
$$

\footnotetext{
${ }^{1}$ For the complete LO evolution equation for $G_{F}\left(x_{1}, x_{2}, \mu\right)$ and $\widetilde{G}_{F}\left(x_{1}, x_{2}, \mu\right)$, see 35.
} 


\begin{tabular}{llll}
\hline \hline \multicolumn{4}{c}{ FIT $1: \chi^{2}=61.47$} \\
\hline$N_{u}^{G}=-0.433$ & $N_{d}^{G}=2.38$ & $N_{s}^{G}=N_{\bar{s}}^{G}=-21.6$ & $N_{\bar{u}}^{G}=N_{\bar{d}}^{G}=-19.4$ \\
$\alpha^{G}=2.14$ & $\beta_{v}^{G}=0.682$ & $\beta_{s}^{G}=-0.956$ & \\
$N_{u}^{F}=1.48$ & $N_{d}^{F}=-2.98$ & $N_{s}^{F}=N_{\bar{s}}^{F}=-46.7$ & $N_{\bar{u}}^{F}=N_{\bar{d}}^{F}=6.76$ \\
$\alpha^{F}=1.40$ & $\beta^{F}=3.52$ \\
\hline \hline \multicolumn{4}{c}{ FIT $2: \chi^{2}=132.9$} \\
\hline$N_{u}^{G}=-2.05$ & $N_{d}^{G}=5.84$ & $N_{s}^{G}=N_{\bar{s}}^{G}=-42.0$ & $N_{\bar{u}}^{G}=N_{\bar{d}}^{G}=-53.8$ \\
$\alpha^{G}=2.59$ & $\beta_{v}^{G}=1.43$ & $\beta_{s}^{G}=0.932$ \\
\hline \hline \multicolumn{4}{c}{ FIT $3: \chi^{2}=64.90$} \\
\hline$N_{u}^{G}=-2.04 \times 10^{6}$ & $N_{d}^{G}=0.237$ & $N_{s}^{G}=-2.71$ \\
$N_{\bar{u}}^{G}=0.443$ & $N_{\bar{d}}^{G}=-4.93$ & $N_{\bar{s}}^{G}=-3.25$ \\
$\alpha_{u}^{G}=4.57$ & $\alpha_{d}^{G}=0.178$ & $\alpha_{s}^{G}=0.641$ \\
$\beta_{u}^{G}=35.4$ & $\beta_{d}^{G}=-0.180$ & $\beta_{s}^{G}=-2.66$ \\
\hline \hline
\end{tabular}

Table 1: Result for the fitting parameters for FIT 1 (SGP+SFP: 13 parameters), FIT 2 (SGP only: Seven parameters) and FIT 3 (SGP only: 12 parameters).

$$
\begin{aligned}
& \alpha_{u, d, s, \bar{u}, \bar{d}, \bar{s}}^{G} \equiv \alpha^{G}, \quad \beta_{u, d}^{G} \equiv \beta_{v}^{G}, \quad \beta_{\bar{u}, \bar{d}, s, \bar{s}}^{G} \equiv \beta_{s}^{G} \\
& N_{u}^{F}, \quad N_{d}^{F}, \quad N_{s}^{F}=N_{\bar{s}}^{F}, \quad N_{\bar{u}}^{F}=N_{\bar{d}}^{F}, \\
& \alpha_{u, d, s, \bar{u}, \bar{d}, \bar{s}}^{F} \equiv \alpha^{F}, \quad \beta_{u, d, s, \bar{u}, \bar{d}, \bar{s}}^{F} \equiv \beta^{F} .
\end{aligned}
$$

FIT 2: Only SGP contribution with seven free parameters:

$$
\begin{aligned}
& N_{u}^{G}, \quad N_{d}^{G}, \quad N_{s}^{G}=N_{\bar{s}}^{G}, \quad N_{\bar{u}}^{G}=N_{\bar{d}}^{G}, \\
& \alpha_{u, d, s, \bar{u}, \bar{d}, \bar{s}}^{G} \equiv \alpha^{G}, \quad \beta_{u, d}^{G} \equiv \beta_{v}^{G}, \quad \beta_{\bar{u}, \bar{d}, s, \bar{s}}^{G} \equiv \beta_{s}^{G} .
\end{aligned}
$$

FIT 3: Only SGP contribution with 12 free parameters:

$$
\begin{aligned}
& N_{u}^{G}, \quad N_{d}^{G}, \quad N_{s}^{G}, \quad N_{\bar{u}}^{G}, \quad N_{\bar{d}}^{G}, \quad N_{\bar{s}}^{G}, \\
& \alpha_{u}^{G}, \quad \alpha_{d}^{G}, \quad \alpha_{s, \bar{u}, \bar{d}, \bar{s}}^{G} \equiv \alpha_{s}^{G}, \quad \beta_{u}^{G}, \quad \beta_{d}^{G}, \quad \beta_{\bar{u}, \bar{d}, s, \bar{s}}^{G} \equiv \beta_{s}^{G} .
\end{aligned}
$$

Several comments are in order for the above constraints among the parameters: With the present limitation of the accumulated SSA data, we could not obtain a convergent set of parameters when we treated all $N_{a}^{G, F}, \alpha_{a}^{G, F}$ and $\beta_{a}^{F, G}$ as independent in the fit with SGP+SFP contributions. We thus introduced the above constraint relation in FIT 1. By allowing reasonable different values for $N_{a}^{G, F}$, we expect to be able to assume $\alpha_{a}^{G, F}$ and $\beta_{a}^{G, F}$ are relatively flavor-independent. This is particularly so for $\alpha_{a}^{G, F}$, since $A_{N}$ rises in the forward direction and is expected not to be very sensitive to $\alpha_{a}^{F, G}$. On the other hand, SGP appears as derivative contribution and thus we assume different values for $\beta_{a}^{G}$ between the valence flavors $(a=u, d)$ and the sea flavors $(a=\bar{u}, \bar{d}, s, \bar{s})$. As for the relations among $N_{a}^{F, G}$ for the "sea" flavors, we have tried all possible combinations among $N_{\bar{u}}^{G, F}= \pm N_{\bar{d}}^{G, F}$ and $N_{s}^{G, F}= \pm N_{\bar{s}}^{G, F}$. It turned out that the above constraint relation in FIT 1 gives the best fit.

We have also performed FIT 2 and FIT 3 with only the SGP contribution in order to see the role of the SFP contribution. FIT 2 assumes the same relation as the SGP contribution in FIT 1 but the SFP contribution is omitted, i.e. FIT 2 has less degrees of freedom compared with FIT 1 by the lack of the SFP contribution. In FIT 3 with the SGP contribution, we allowed more freedom in the parameters. This fitting contains the Fit II of [26] (hereafter, simply referred to as KQVY06) as a special case as well as the above FIT 2. With this maximally allowed freedom in the SGP contribution, the number of degrees of freedom in FIT 3 is almost the same as FIT 1 and thus one can see whether the SFP contribution can be replaced by an appropriate choice of the SGP contribution.

The results of the fitting in the above three cases are shown in Table 1. The calculated $A_{N}$ are also shown in Fig. 1 together with the RHIC data. For comparison, we have also shown in the same 


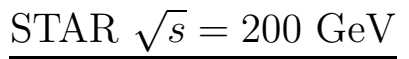
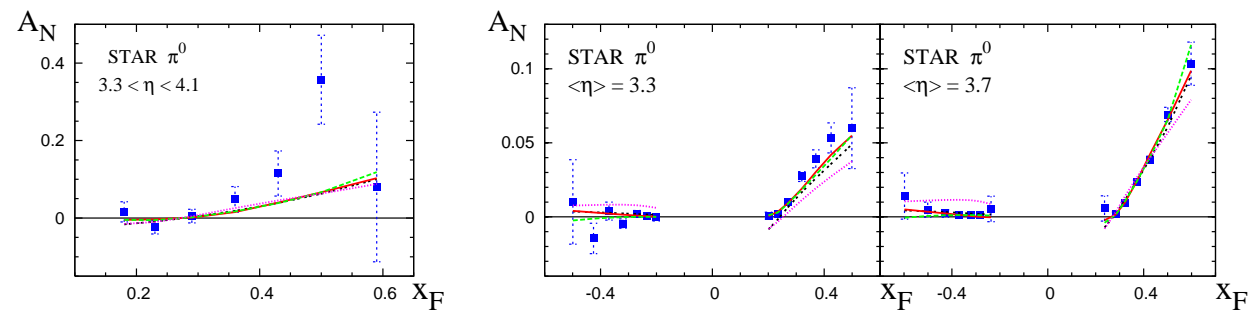

$\underline{\text { BRAHMS } \sqrt{s}=62.4 \mathrm{GeV}}$
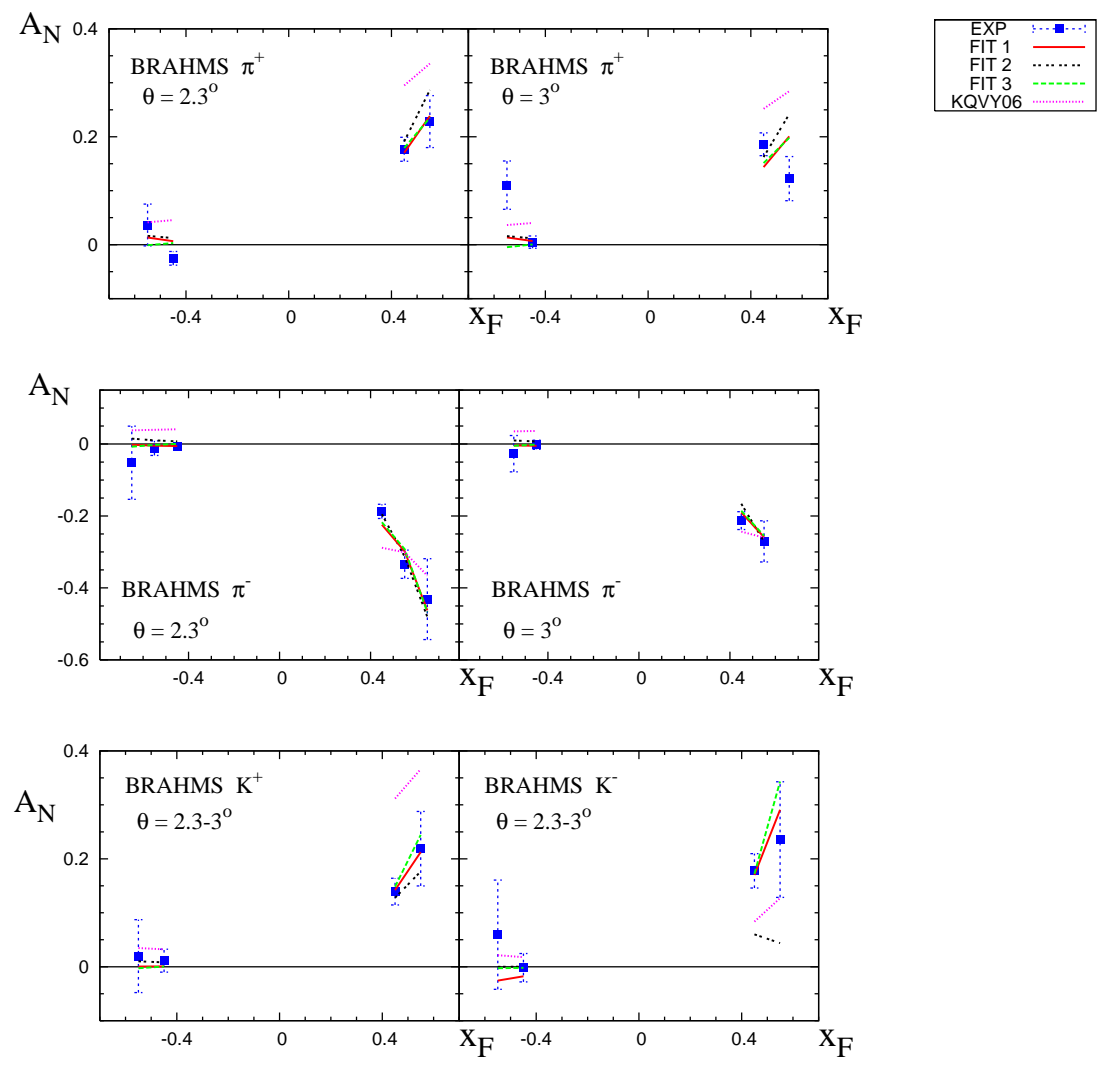

Figure 1: The results of the three fits for $A_{N}$. The solid lines are for FIT 1, the long-dotted lines are for FIT 2, and the dashed lines are for FIT 3. The result by KQVY06 is also shown by the short-dotted lines. 

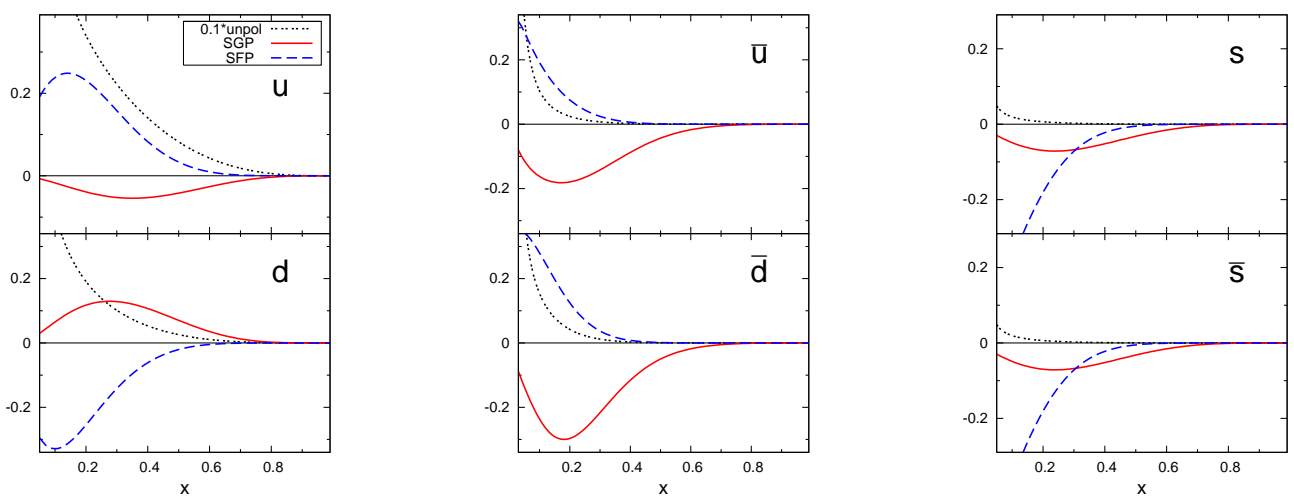

Figure 2: The SGP function $G_{F}^{a}(x, x)$ and the SFP function $G_{F}^{a}(0, x)+\widetilde{G}_{F}^{a}(0, x)$ at $Q^{2}=2.4 \mathrm{GeV}^{2}$ for each quark-flavor obtained in FIT 1 in comparison to the unpolarized quark distribution $f_{a}(x)$ [scaled by factor $1 / 10]$.
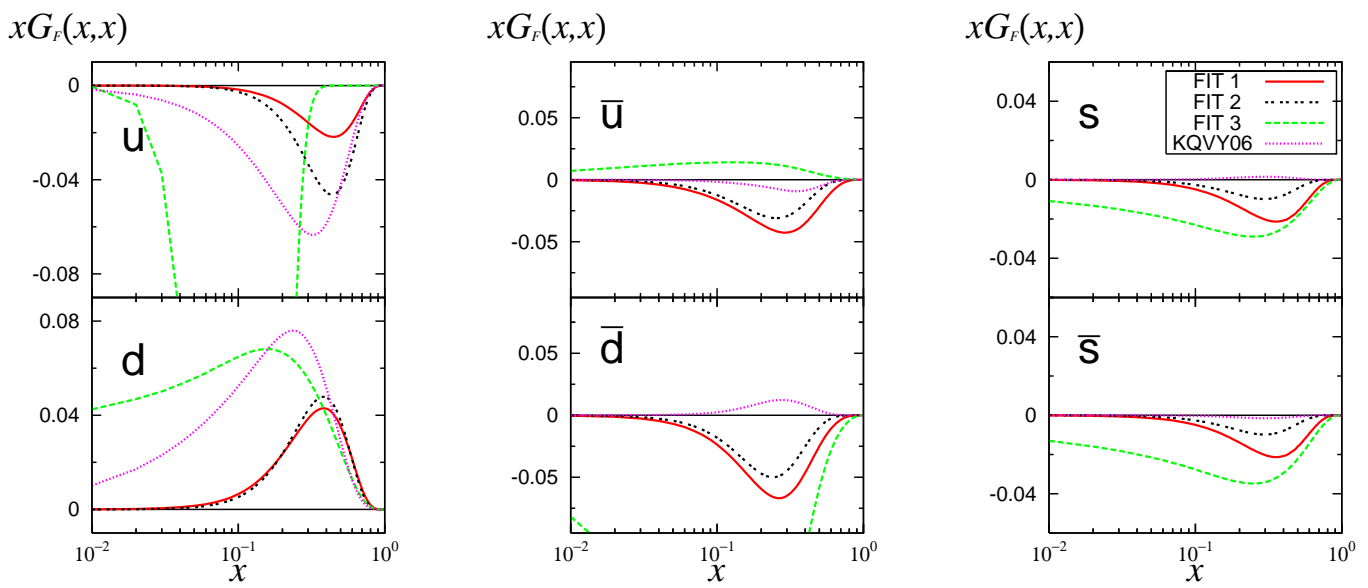

Figure 3: SGP functions at $Q^{2}=2.4 \mathrm{GeV}^{2}$ obtained in three fits. The SGP function in KQVY06 is also shown by using the relation $G_{F}^{a}(x, x)=-\frac{\sqrt{4 \pi \alpha_{s}}}{\pi M_{N}} T_{F, a}(x, x)$ for each quark flavor. For antiquark flavors, the sign of this relation is opposite.

figure the result of KQVY06 [26] obtained with the CTEQ5L distribution function 38] and the Kretzer's fragmentation function [39. From Fig. 1, one sees that FIT 1 and FIT 3 reproduce $A_{N}$ data for all mesons, while FIT 2 and KQVY06 fails for $K^{-}$. We note that our FIT 3 has converged into a result that is different from KQVY06. This is because we have used different sets of data from KQVY06. The deviation of the curves of KQVY06 from data for the charged mesons is also due to the difference of the used data sets. (Also note the kinematics of [7] differs from Fig. 10 of [26.) This feature is also represented in the resulting $\chi^{2}$, which are $61.47,132.9$ and 64.90 for FIT 1, FIT 2, and FIT 3, respectively. Although FIT 3 reproduces $A_{N}$ equally well as FIT 1, the behavior of the obtained fitting functions are extreme and looks unphysical as is seen by the resulting parameters in the functions shown in Table 1. This suggests that the SFP contribution is also a necessary source for SSA and plays an important role which cannot be substituted by the SGP contribution.

Figure 2 shows the obtained SGP and SFP functions in FIT 1 for each quark flavor in comparison with the unpolarized parton density (scaled by factor 1/10). One sees that the SGP and SFP functions are comparable in magnitude, but the SFP functions are more shifted in smaller $x$ regions. Among SGP functions, those for the "valence" flavors ( $u$ and $d$ quarks) spread more into a larger $x$ region than those for sea flavors. This spreading into a larger $x$ region actually plays an important role for the rising $A_{N}$ in the forward region. We observe that the flavor dependence of the SFP functions also has a similar tendency. Owing to the behaviors of each function, the largest contribution to $A_{N}^{\pi^{+}}$and $A_{N}^{\pi^{-}}$are, 
respectively, from $u$ and $d$ quark SGP functions. As for the kaons, the SGP contribution from strange quark turned out to be the largest with small corrections from other flavors and the SFP contributions. This is largely due to the large strangeness component in the DSS fragmentation function. From the definition of the SGP and SFP functions, the latter might be interpreted as a more "exotic" component of the correlations, while the former may be closer to a quark parton density accompanied by the soft gluon. This may lead to the SFP function to have nonzero values only in the smaller $x$ region.

To compare the functions in FIT 1 with other cases, we have shown in Fig. 3 the SGP functions obtained in each fit. For comparison we have also shown the SGP functions of KQVY06. As is seen from Fig. 3, the SGP functions for $u$ and $\bar{d}$ quarks in FIT 3 are huge compared with those for other quarkflavors, and the former is sharply peaked, which are the result of the extreme values of the parameters in FIT 3 shown in Table 1. This unphysical behavior of the SGP function can be taken as a manifestation of the fact that the SGP function alone can not reproduce all of the observed $A_{N}$, and other sources of SSA are necessary. From Fig. 3, the SGP functions in FIT 1 turn out to be smaller compared with those in FIT 2 and FIT 3 , since the SFP functions can give rise to the additional $A_{N}$ s. Moderate behaviors of the SGP and SFP functions given in FIT 1 as shown in Figs. 2 and 3 indicate that the SFP contribution is a natural source of $A_{N}$ together with the SGP contribution.

To see the relative magnitude of the SFP contributions in FIT 1, we have shown in Fig. 4 the decomposition of $A_{N}$ into the SGP and SFP contributions. One sees from the figure that in many cases the SGP brings a larger contribution as a whole, while the SFP works as a small but non-negligible correction. For the kaons, we observed that the SFP contribution from $u$ and $d$ quarks are quite large but they tend to cancel each other. As one can see from (6) and (14) the SFP hard cross sections are larger than the SGP hard cross section typically by factor $N_{c}^{2} \underline{30}$. However, the SGP functions spread more in the larger $x$ region as shown in Fig. 2 and also contribute as a derivative. Therefore it brings a large contribution to $A_{N}$ in the forward region.

Figure 5 shows the $P_{h T}$ dependence of $A_{N}^{\pi^{0}}$ for $\sqrt{S}=200 \mathrm{GeV}$ together with the STAR data. All fits give similar curves for each $x_{F}$ and approximately reproduce experimental data. This is quite natural because our three fits used correct $P_{h T}$ at each $x_{F}$ of the $A_{N}$ data shown in Fig. 1. If we had included E704 data in the fit without taking into account of the $P_{h T}$ dependence, deviation from the RHIC data may have been observed in the $P_{h T}$-depndence as was the case in the analysis by KQVY06.

Using the SFP and SGP functions in the fits, we have calculated $A_{N}^{\pi}$ at the FNAL energy $\sqrt{S}=20$ $\mathrm{GeV}$ for $p^{\uparrow} p \rightarrow \pi X$ and $\bar{p}^{\uparrow} p \rightarrow \pi X$. As mentioned before, we did not include the $A_{N}$ data of FNAL-E704 $(\sqrt{S}=20 \mathrm{GeV})$ in the fitting. We do this comparison as a semiquantitative test of our fit result in terms of the twist-3 formalism at a lower energy. With our convention for the twist-3 distributions for the "antiquark" flavor in (5), those in the antiproton are related to those in the proton as

$$
\left.G_{F}^{a}\left(x_{1}, x_{2}\right)\right|_{\text {anti-proton }}=\left.G_{F}^{\bar{a}}\left(x_{1}, x_{2}\right)\right|_{\text {proton }},\left.\quad \widetilde{G}_{F}^{a}\left(x_{1}, x_{2}\right)\right|_{\text {anti-proton }}=\left.\widetilde{G}_{F}^{\bar{a}}\left(x_{1}, x_{2}\right)\right|_{\text {proton }} .
$$

The result was shown in Fig. 6 together with the E704 data [1]. From Fig. 6, one sees that FIT 1 and FIT 2 give qualitatively similar curves as the data, while FIT 3 shows the deviation from the data at small- $x_{F}$ for $\pi^{0}$. As pointed out in [34, the NLO QCD in the collinear factorization can not reproduce the unpolarized cross section at the fixed target energy and thus our twist-3 formula for $A_{N}$ may not be directly used for the FNAL data. With this in mind, KQVY06 included the E704 data in their fitting by multiplying the ad hoc factor $N_{\mathrm{E} 704}=1 / 2$ to the theory calculation. If we had known this $N_{\mathrm{E} 704}$ more precisely, we could have included the E704 data in our analysis, which is beyond the scope of the present study. From Fig. 6, one sees the E704 data is smaller than the calculated $A_{N}$, which is consistent with the analysis in $[26$.

\section{Summary}

In this paper, we have presented a numerical analysis of the RHIC $A_{N}$ data for $p^{\uparrow} p \rightarrow h X(h=\pi, K)$ in terms of the twist-3 mechanism in the collinear factorization. As a source of the asymmetry we have included all the contribution from the twist-3 quark-gluon correlation functions in the transversely polarized nucleon, which consist of the SGP and SFP contributions. We have shown that the combination of the SGP and SFP contributions can reproduce the $A_{N}$ data at RHIC with moderate choice of the functions, while it is difficult to reproduce all of them if one keeps only the SGP contribution. In particular, $A_{N}$ for $K^{ \pm}$was properly reproduced by the inclusion of the SFP contribution. Although the 
$\operatorname{STAR} \sqrt{s}=200 \mathrm{GeV}$
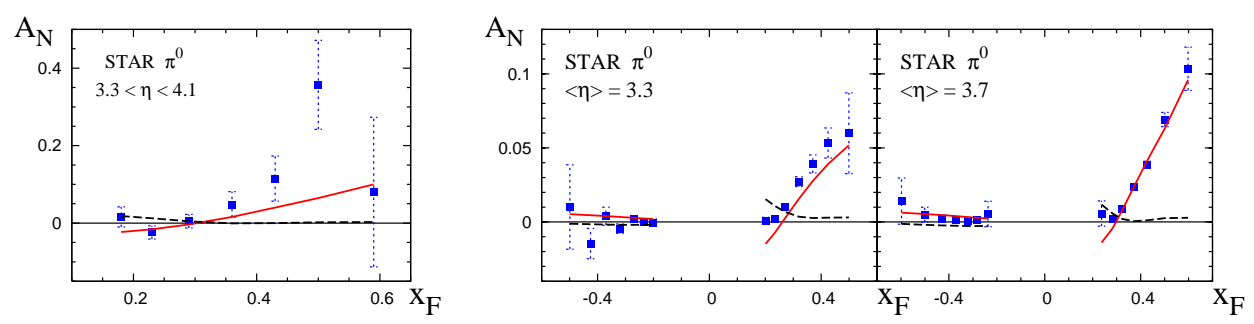

BRAHMS $\sqrt{s}=62.4 \mathrm{GeV}$
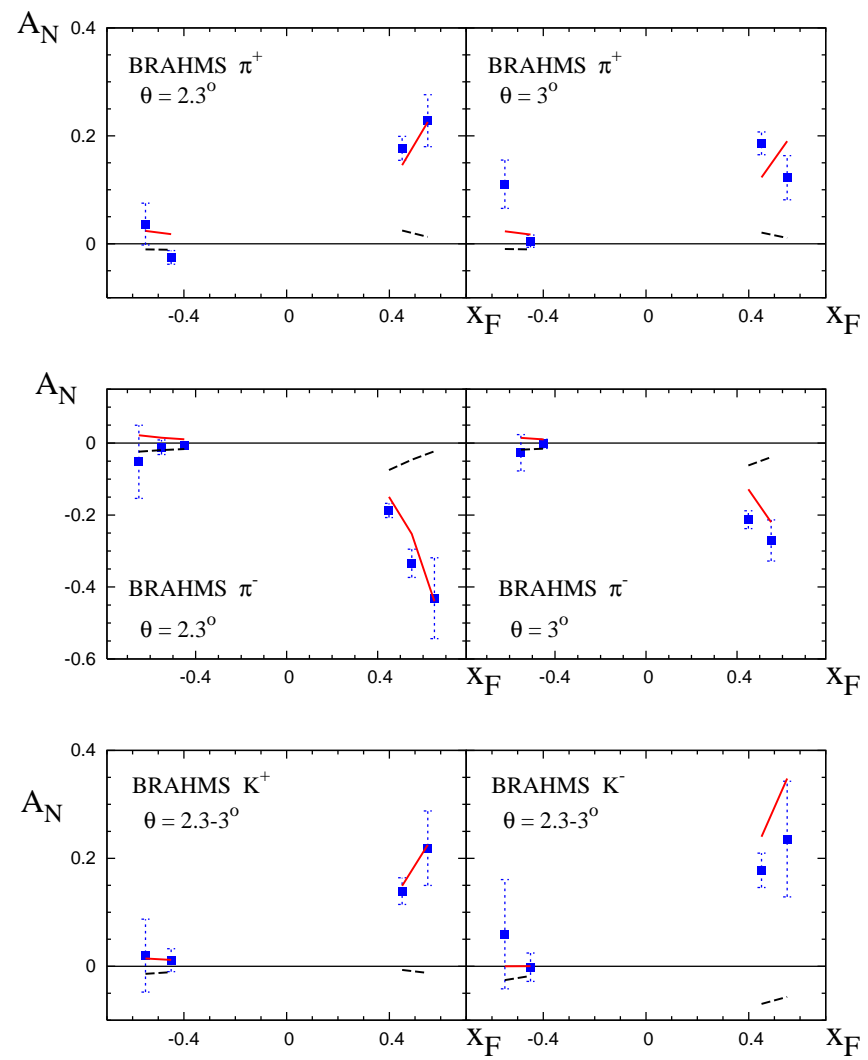

Figure 4: Separation of $A_{N}$ in FIT 1 into the SGP (solid lines) and the SFP (dashed lines) contributions. 


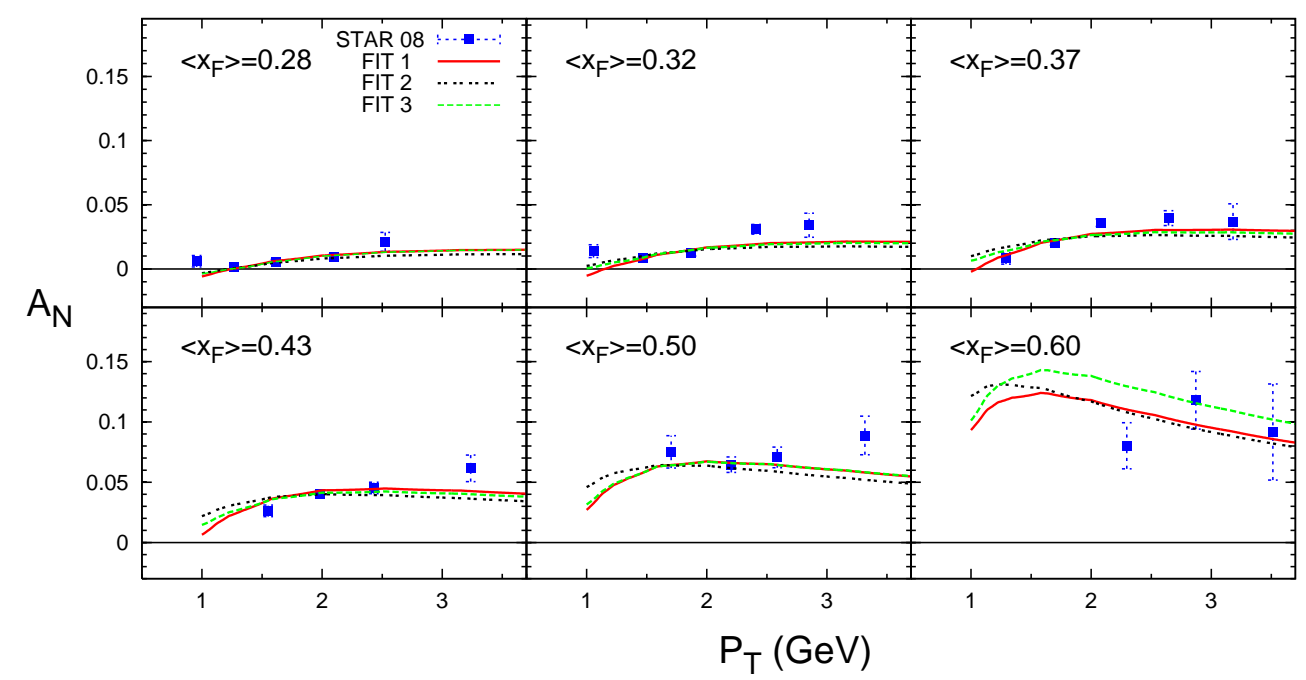

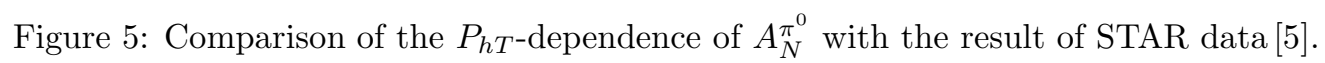

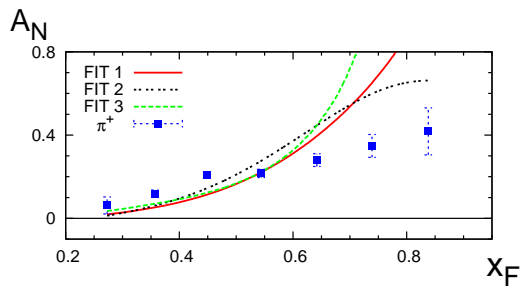

$A_{N}$

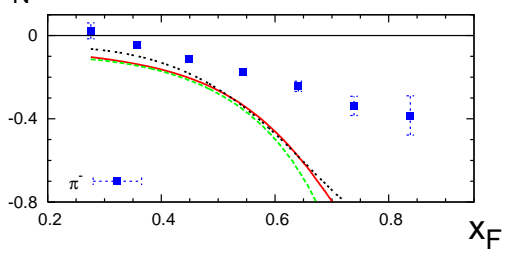

$A_{N}$

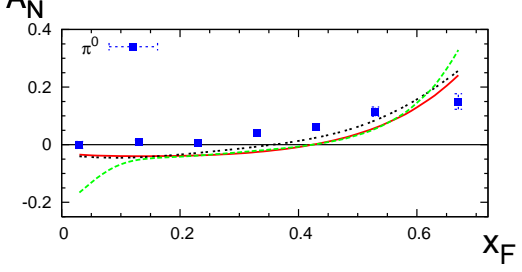

$\mathrm{A}_{\mathrm{N}}$

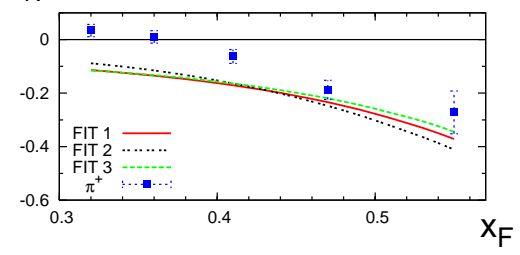

$A_{N}$

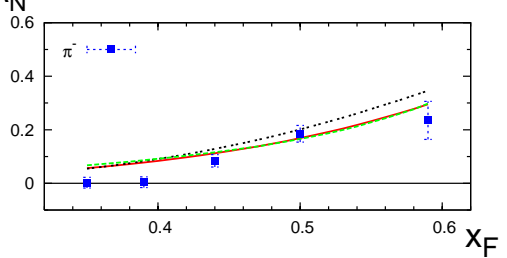

$A_{N}$

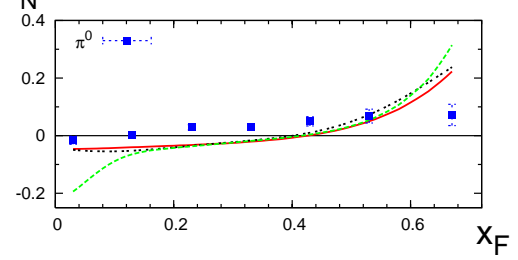

Figure 6: Comparison of the fits with the FNAL-E704 data. We set $P_{h T}=1.2(\mathrm{GeV})$ in the theory calculation. The left three figures are for the $p p$ collision, and the right three figures are for the $\bar{p} p$ collision. 
relevance of the SFP contribution is numerically supported, we do not have any dynamical information on the shape and magnitude of the SGP and SFP functions at this stage. 2 In addition, our present analysis did not include the second term of (2), which could be another important source of SSA. To clarify these points, one needs more variety of SSA data together with the nonperturbative study on the SGP and SFP functions.

\section{Acknowledgement}

We thank D. de Florian for providing us with the Fortran code of their fragmentation function.

\footnotetext{
${ }^{2}$ There is, however, a model calculation [40] showing the SFP function is small.
} 


\section{References}

[1] D.L. Adams et al. (E704 Collaboration), Phys. Lett. B261, 201 (1991); 264, 462 (1991).

[2] V.Y. Alexakhin et al.(COMPASS Collaboration), Phys. Rev. Lett 94, 202002 (2005); E.S. Ageev et al. (COMPASS Collaboration), Nucl. Phys. B765, 31 (2007); M. Alekseev et al. (COMPASS Collaboration), Phys. Lett. B673, 127 (2009).

[3] A. Airapetian et al. (HERMES Collaboration), Phys. Rev. D64, 097101 (2001); Phys. Rev. Lett. 94, 012002 (2005); 103, 152002 (2009).

[4] J. Adams et al. (STAR Collaboration), Phys. Rev. Lett. 92, 171801 (2004).

[5] B.I. Abelev et al. (STAR Collaboration), Phys. Rev. Lett. 101, 222001 (2008).

[6] S. S. Adler et al. (PHENIX Collaboration), Phys. Rev. Lett. 95, 202001 (2005).

[7] I. Arsene et al. (BRAHMS Collaboration), Phys. Rev. Lett. 101, 042001 (2008).

[8] G.L. Kane, J. Pumplin, and W. Repko, Phys. Rev. Lett. 41, 1689 (1978).

[9] D. W. Sivers, Phys. Rev. D41, 83 (1990); Phys. Rev. D43, 261 (1991).

[10] J. C. Collins, Nucl. Phys. B396, 161 (1993).

[11] J. C. Collins, Phys. Lett. B536, 43 (2002).

[12] A. V. Belitsky, X. D. Ji, and F. Yuan, Nucl. Phys. B656, 165 (2003).

[13] D. Boer, P. Mulders and F. Pijlman, Nucl. Phys. B667, 201 (2003).

[14] J. C. Collins and A. Metz, Phys. Rev. Lett. 93, 252001 (2004).

[15] S. Meissner and A.Metz, Phys. Rev. Lett. 102, 172003 (2009).

[16] J.C. Collins and D.E. Soper, Nucl. Phys. B193, 381 (1981); B213, 545(E) (1983).

[17] J.C. Collins, D.E. Soper, and G. Sterman, Nucl. Phys. B250, 199 (1985).

[18] X. D. Ji, J. P. Ma, and F. Yuan, Phys. Rev. D71, 034005 (2005); Phys. Lett. B597, 299 (2004).

[19] C. J. Bomhof, P. J. Mulders, and F. Pijlman, Phys. Lett. B596, 277 (2004); Eur. Phys. J. C47, $147(2006)$;

A. Bacchetta, C. J. Bomhof, P. J. Mulders, and F. Pijlman, Phys. Rev. D72, 034030 (2005);

C.J. Bomhof and P.J. Mulders, JHEP 0702:029, (2007);

C.J. Bomhof and P.J. Mulders, Nucl. Phys. B795, 409 (2008).

[20] M. Anselmino, M. Boglione, U. D'Alesio, A. Kotzinian, F. Murgia, and A. Prokudin, Phys. Rev. D71, 074006 (2005);

M. Anselmino, M. Boglione, U. D’Alesio, A. Kotzinian, F. Murgia, A. Prokudin, and C.Türk, Phys. Rev. D75, 054032 (2007);

M. Anselmino, M. Boglione, U. D’Alesio, A. Kotzinian, S. Melis, F. Murgia, A. Prokudin, and C. Türk, Eur. Phys. J. A39, 89 (2009);

and references quoted therein.

[21] A.V. Efremov and O.V. Teryaev, Sov. J. Nucl. Phys. 36, 140 (1982), Yad. Fiz. 36, 242 (1982);

A.V. Efremov and O.V. Teryaev, Phys. Lett. B150, 383 (1985).

[22] J.W. Qiu and G. Sterman, Nucl. Phys. B378, 52 (1992).

[23] J.W. Qiu and G. Sterman, Phys. Rev. D59, 014004 (1998). 
[24] Y. Kanazawa and Y. Koike, Phys. Lett. B478, 121 (2000);

Y. Kanazawa and Y. Koike, Phys. Lett. B490, 99 (2000).

[25] H. Eguchi, Y. Koike, and K. Tanaka, Nucl. Phys. B752, 1 (2006);

H. Eguchi, Y. Koike, and K. Tanaka, Nucl. Phys. B763, 198 (2007).

[26] C. Kouvaris, J.W. Qiu, W. Vogelsang, and F. Yuan, Phys. Rev. D74, 114013 (2006).

[27] Y. Koike and K. Tanaka, Phys. Lett. B 646, 232 (2007) [Erratum-ibid. B 668, 458 (2008)]

[28] Y. Koike and K. Tanaka, Phys. Rev. D76, 011502(R) (2007).

[29] Z. B. Kang and J. W. Qiu, Phys. Rev. D 78, 034005 (2008).

Z. B. Kang, J. W. Qiu, W. Vogelsang, and F. Yuan, Phys. Rev. D78 114013 (2008).

[30] Y. Koike and T. Tomita, Phys. Lett. B675, 181 (2009).

[31] X.D. Ji, J.W. Qiu, W. Vogelsang, and F. Yuan, Phys. Lett. 97, 082002 (2006);

X.D. Ji, J.W. Qiu, W. Vogelsang, and F. Yuan, Phys. Rev. D73, 094017 (2006);

X.D. Ji, J.W. Qiu, W. Vogelsang, and F.Yuan, Phys. Lett. B638, 178 (2006);

Y. Koike, W. Vogelsang, and F. Yuan, Phys. Lett. B659, 878 (2008).

[32] F. Yuan and J. Zhou, Phys. Rev. Lett. 103, 052001 (2009);

Z. Kang, F. Yuan, and J. Zhou, arXiv:1002.0399: [hep-ph].

[33] B.L. Combridge, J. Kripfganz, and J. Ranft, Phys. Lett. B70, 234 (1977);

R. Cutler and D.W. Sivers, Phys. Rev. D17, 196 (1978);

J.F. Owens, E. Reya, and M. Glück, Phys Rev. D18, 1501 (1978).

[34] C. Bourrely and J. Soffer, Eur. Phys. J. C36, 371 (2004).

[35] V. M. Braun, A. N. Manashov, and B. Pirnay, Phys. Rev. D 80, 114002 (2009).

[36] M. Glück, E. Reya, and A. Vogt, Eur. Phys. J. C5, 461 (1998)

[37] D. de Florian, R. Sassot, and M. Stratmann, Phys. Rev. D75, 114010 (2007).

[38] H.L. Lai et al. (CTEQ Collaboration), Eur. Phys. J. C12, 375 (2000).

[39] S. Kretzer, Phys. Rev. D62, 054001 (2000).

[40] Z. B. Kang, J. W. Qiu and H. Zhang, arXiv:1004.4183 [hep-ph]. 

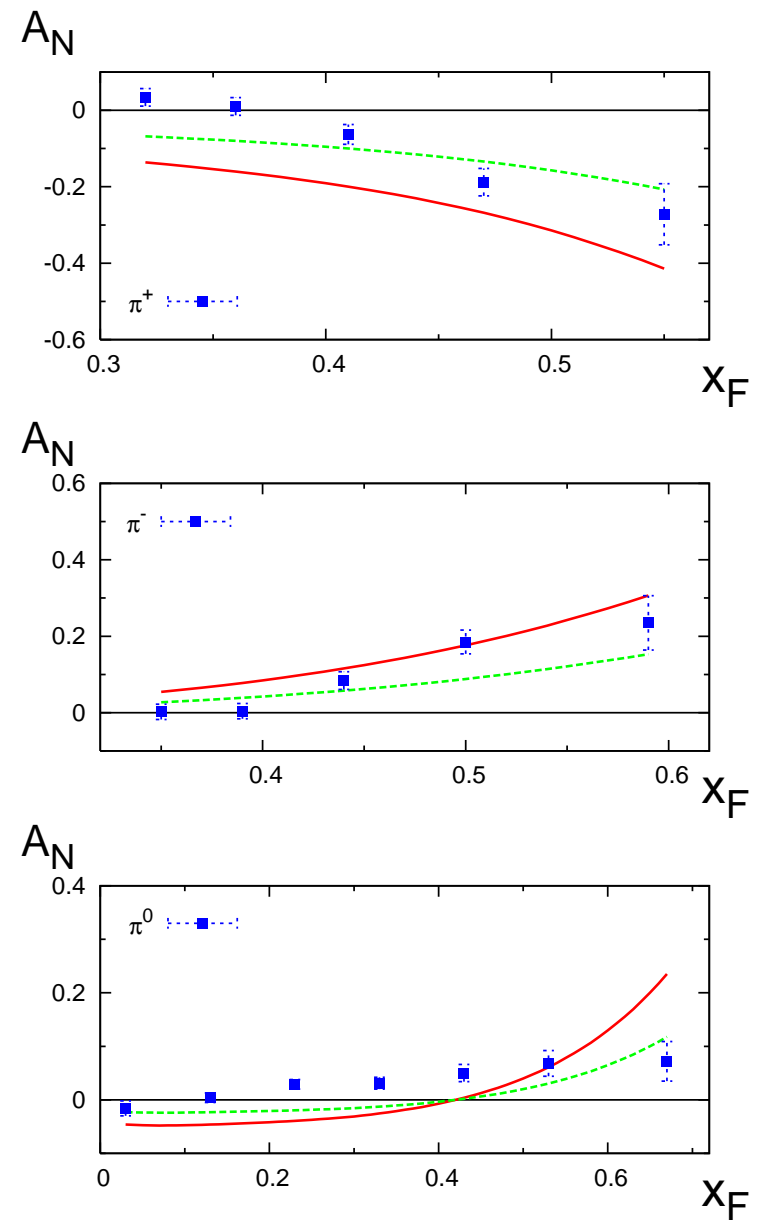


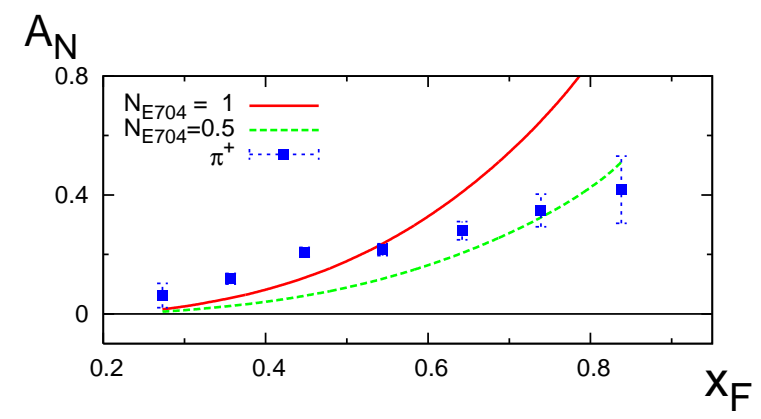

$A_{N}$

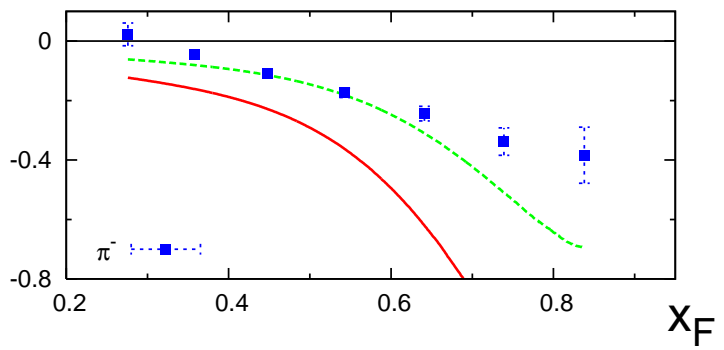

$A_{N}$

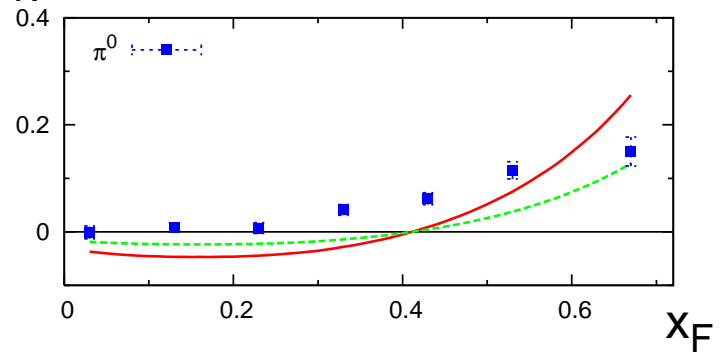




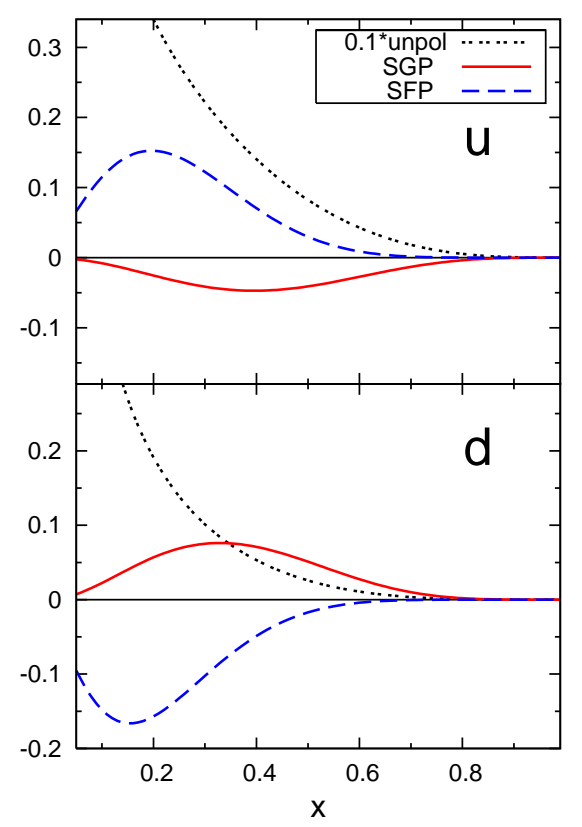




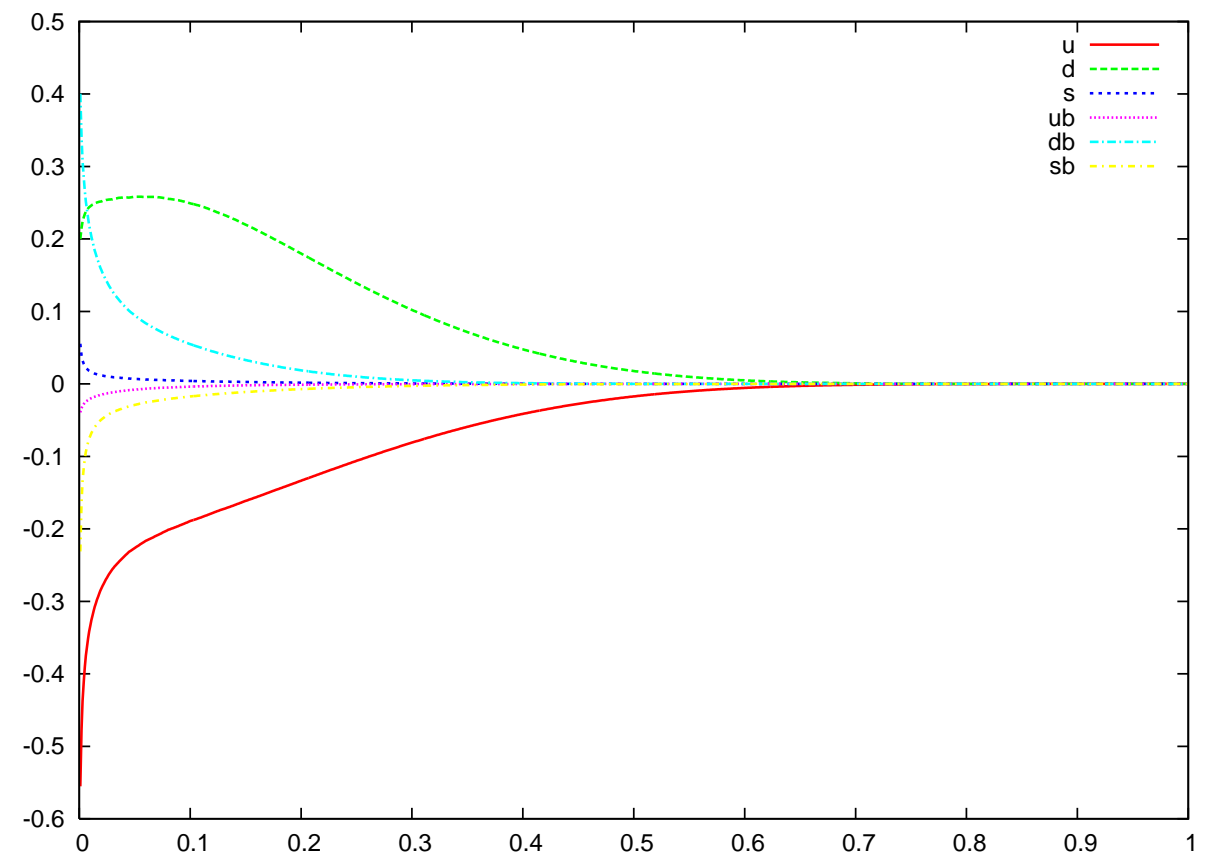

